\title{
Impact of Immune-Related Adverse Events on Efficacy of Immune Checkpoint Inhibitors in Patients with Advanced Hepatocellular Carcinoma
}

\author{
Kennedy Yao Yi Ng ${ }^{a}$ Sze Huey Tan ${ }^{b, c}$ Jack Jie En Tan ${ }^{d}$ Desiree Shu Hui Tay ${ }^{d}$ \\ Ailica Wan Xin Lee ${ }^{d}$ Andrea Jing Shi Ang ${ }^{e}$ Lawrence Wen Jun Wong ${ }^{f}$ \\ Su Pin Choo ${ }^{a, g}$ David Wai-Meng Tai ${ }^{a, c}$ Joycelyn Jie Xin Lee ${ }^{a, c}$ \\ aDivision of Medical Oncology, National Cancer Centre Singapore, Singapore, Singapore; bivision of Clinical Trials \\ and Epidemiological Sciences, National Cancer Centre Singapore, Singapore, Singapore; 'Oncology Academic \\ Program, Duke-NUS Medical School, Singapore, Singapore; ${ }^{d}$ Yong Loo Lin School of Medicine, National University of \\ Singapore, Singapore, Singapore; 'Division of Internal Medicine, Singapore General Hospital, Singapore, Singapore; \\ fDivision of Pediatrics, KK Women and Children's Hospital, Singapore, Singapore; ' $\mathrm{C}$ urie Oncology, Singapore, \\ Singapore
}

\section{Keywords}

Hepatocellular carcinoma $\cdot$ Immune checkpoint inhibitors · Immune-related adverse events · Survival · Response

\begin{abstract}
Introduction: Development of immune-related adverse events (irAEs) has been associated with enhanced efficacy with the use of immune checkpoint inhibitors (ICls). It remains unknown whether such an association exists in advanced hepatocellular carcinoma (aHCC). This study aims to evaluate the association between irAEs and ICl efficacy in patients with aHCC. Methods: We performed a retrospective cohort study on patients with aHCC who received at least one dose of an ICl between May 2015 and November 2019 at the National Cancer Centre Singapore. The primary study objectives were to compare the overall survival (OS) and progression-free survival (PFS) between patients with and without irAEs. Complementary multivariable landmark analyses were performed at the 6-week and 12-week landmarks. Data
\end{abstract}

karger@karger.com www.karger.com/lic

Karger"

BOPEN ACCESS
(C) 2021 The Author(s)

Published by S. Karger AG, Basel

This is an Open Access article licensed under the Creative Commons Attribution-NonCommercial-4.0 International License (CC BY-NC) (http://www.karger.com/Services/OpenAccessLicense), applicable to the online version of the article only. Usage and distribution for commercial purposes requires written permission. cutoff was December 31, 2020. Results: One hundred and sixty-eight patients were included. Median age was 69 years, $85.7 \%$ were male, $57.7 \%$ had hepatitis B infection, $60.7 \%$ had ECOG 0, and $78.0 \%$ had Child-Pugh A liver cirrhosis. $82.7 \%$ received $\mathrm{ICI}$ monotherapy, while $17.3 \%$ received $\mathrm{ICI}$ in combination. Development and severity of irAE were correlated with survival. The median PFS for grade $\geq 3$ irAE versus grades $1-2$ irAE versus no irAE was 8.5 versus 3.6 versus 1.3 mths $(p<0.001)$. The median OS for grade $\geq 3$ irAE versus grades $1-2$ irAE versus no irAE was 26.9 versus 14.0 versus $4.6 \mathrm{mths}$ $(p<0.001)$. Patients with $\geq 2$ irAEs had a significantly longer OS on multivariable analysis (adjusted hazard ratio [aHR] 0.35, $p<0.001$ ). The presence of grade $\geq 3$ irAEs was associated with a significantly longer OS on the multivariable analysis at the 6-week landmark (aHR0.34, $p=0.030$ ) and 12-week landmark (aHR0.28, $p=0.011$ ). The use of systemic corticosteroids in patients with irAE was associated with a trend toward a longer OS (20.7 vs. $14.3 \mathrm{mths}, p=0.064)$. Conclusion:

Kennedy Yao Yi Ng and Sze Huey Tan contributed equally.
Correspondence to:

Joycelyn Jie Xin Lee, joycelyn.lee.j.x@ singhealth.com.sg 
Our study suggests that the presence of all-grade irAEs may be a potential prognostic biomarker in patients with aHCC treated with $\mathrm{ICl}$. Patients with more severe irAEs and multisystem involvement have better prognosis. The prompt use of systemic corticosteroids to treat patients with irAEs is key to ensure the best long-term outcomes for these patients.

(C) 2021 The Author(s).

Published by S. Karger AG, Basel

\section{Introduction}

Hepatocellular carcinoma (HCC) is a highly lethal disease, being the sixth most common cancer and second most common cause of cancer-related death globally [1]. HCC can be treated with surgical, locoregional, or systemic treatment options. Most patients with HCC are diagnosed in the advanced stage, where systemic therapy options are the mainstay of treatment. Previously, the first-line treatment of advanced HCC (aHCC) was limited to tyrosine kinase inhibitors (TKIs) sorafenib or lenvatinib $[2,3]$. In recent years, immune checkpoint inhibitors (ICIs) have been found to be efficacious. The combination of atezolizumab and bevacizumab was found in the phase 3 randomized clinical trial (RCT) IMbrave150 to be superior to sorafenib in terms of overall survival (OS) and is now a standard of care in the first-line treatment of aHCC [4].

By upregulating the immune system, immunotherapy can result in inflammatory side effects, which are collectively referred to as immune-related adverse events (irAEs). These are defined as side effects with potential immunological basis, which requires more frequent monitoring and possible treatment with systemic steroids [5]. Single-agent nivolumab or pembrolizumab in the phase 3 randomized clinical trials CheckMate- 459 and Keynote-240, respectively, resulted in $2-28 \%$ all-grade irAEs. Twenty-two percent of patients developed grade $\geq 3$ treatment-related adverse events (trAEs) with nivolumab, while $7.2 \%$ developed grade $\geq 3$ trAEs with pembrolizumab $[6,7]$. In IMbrave150 combination atezolizumab and bevacizumab, $68.7 \%$ and $25.8 \%$ had allgrade irAEs and grade $\geq 3$ irAEs, respectively. In CheckMate-040, patients receiving combination nivolumab and ipilimumab saw rash being the most common allgrade irAE and hepatitis being the most common grade $\geq 3$ irAE with incidence of $35 \%$ and $20 \%$, respectively [8].

It has been postulated that there is a possible association between irAEs with improved clinical outcomes, given that the two are thought to have similar immunological basis. Recent studies have demonstrated this positive association in patients treated with ICI with other solid malignancies, namely advanced melanoma [9], urothelial cancer [10], renal cell carcinoma [11], non-small cell lung cancer (NSCLC) [12], gastric cancer [13], and other gastrointestinal cancers (which includes a small cohort of 32 patients with aHCC) [14]. However, the data are conflicting as there are also studies demonstrating no such associations $[15,16]$. It is also currently unknown if the severity of irAE, particular sites of irAE or number of organ systems involved (i.e., multisystem irAE), has an association with the efficacy of ICI.

In addition, irAEs are often treated with systemic corticosteroids. Concerns have been raised that their use has a detrimental effect on outcome, as it counteracts the effects of immunostimulatory drugs. A meta-analysis and systematic review demonstrated a negative association between the use of systemic corticosteroids and clinical outcomes, though the studies included consisted mainly of patients with lung cancer and melanoma with no patients with HCC included [17]. This study aims to describe the incidence, time to onset and predictors of irAE, their association with survival and efficacy of ICI in patients with aHCC, and if the use of systemic corticosteroids for treatment of irAE is associated with reduced efficacy of ICI.

\section{Methods}

Study Design

We performed a retrospective cohort study on all patients with aHCC at the National Cancer Centre Singapore who received at least one dose of an ICI between May 2015 and November 2019. Retrospective chart review was performed to retrieve patient and disease characteristics as well as treatment response data. The data cutoff date was December 31, 2020. Data were de-identified for all statistical analyses.

The patients were categorized into several groups based on the variable of interest studied in relation to clinical outcomes. Firstly, they were categorized based on the incidence of irAEs: those with irAEs (irAE group) or those without (non-irAE group). irAEs were defined as adverse effects with potential immunologic basis. These were graded using the Common Terminology Criteria for Adverse Events v4.03 criteria [18]. irAEs were defined by the treating oncologist by excluding alternative diagnosis and assessing if there was clinical improvement with irAE-based treatment $[19,20]$. In addition, another oncologist reviewed the clinical charts of all patients in this study to determine if the AEs are attributable to the immunotherapy. Patients with irAEs were further subdivided based on whether they received treatment with systemic steroids. The starting dose, duration, number of courses of systemic steroids, and usage of adjunctive immunosuppressants were collected. Patients with multiple irAEs were classified as having concurrent irAEs if the irAEs affecting different systems occurred within 2 weeks apart and classified as sequential irAEs if the irAEs oc-
10

Liver Cancer 2022;11:9-21 DOI: $10.1159 / 000518619$
Ng/Tan/Tan/Tay/Lee/Ang/Wong/Choo/ Tai/Lee 
Table 1. Demographics of patients without irAE and with irAE

\begin{tabular}{|c|c|c|c|c|}
\hline & \multirow{2}{*}{$\begin{array}{l}\text { Total } \\
(n=168)\end{array}$} & \multicolumn{3}{|l|}{ Frequency (\%) } \\
\hline & & without irAE $(n=71)(42.3)$ & with irAE $(n=97)(57.7)$ & $p$ value \\
\hline \multicolumn{5}{|l|}{ Age } \\
\hline Mean (SD) & $67(11.0)$ & $65(11.3)$ & $69(10.6)$ & \multirow{3}{*}{$0.004^{\#}$} \\
\hline Median (IQR) & $69(60,75)$ & $66(57,73)$ & $71(63,76)$ & \\
\hline Range & $25-88$ & $33-88$ & $25-85$ & \\
\hline \multicolumn{5}{|l|}{ Gender } \\
\hline Male & $144(85.7)$ & $56(78.9)$ & $88(90.7)$ & \multirow{2}{*}{0.030} \\
\hline Female & $24(14.3)$ & $15(21.1)$ & $9(9.3)$ & \\
\hline \multicolumn{5}{|l|}{ Ethnicity } \\
\hline Chinese & $114(67.9)$ & $48(67.6)$ & $66(68.0)$ & \multirow{5}{*}{$0.487 \wedge$} \\
\hline Malay & $10(6.0)$ & $3(4.2)$ & $7(7.2)$ & \\
\hline Indian & $4(2.4)$ & $1(1.4)$ & $3(3.1)$ & \\
\hline Caucasian & $2(1.2)$ & $2(2.8)$ & $0(0.0)$ & \\
\hline Others & $38(22.6)$ & $17(23.9)$ & $21(21.6)$ & \\
\hline \multicolumn{5}{|l|}{ BMI } \\
\hline Mean (SD) & $24(4.1)$ & $25(4.0)$ & $24(4.2)$ & \multirow{3}{*}{$0.510^{\#}$} \\
\hline Median (IQR) & $24(22,26)$ & $24(22,27)$ & $24(22,26)$ & \\
\hline Range & $15-39$ & $17-36$ & $15-39$ & \\
\hline \multicolumn{5}{|l|}{ BMI } \\
\hline $\mathrm{BMI}<23$ & $64(38.1)$ & $25(35.2)$ & $39(40.2)$ & \multirow{3}{*}{$\begin{array}{l}0.602 \wedge \\
(0.568)\end{array}$} \\
\hline $\mathrm{BMI} \geq 23$ & $101(60.1)$ & $44(62.0)$ & $57(58.8)$ & \\
\hline Unknown & $3(1.8)$ & $2(2.8)$ & $1(1.0)$ & \\
\hline \multicolumn{5}{|l|}{ ECOG status } \\
\hline ECOG 0 & $102(60.7)$ & $38(53.5)$ & $64(66.0)$ & \multirow{3}{*}{$0.037^{\wedge}$} \\
\hline ECOG 1 & $59(35.1)$ & $27(38.0)$ & $32(33.0)$ & \\
\hline ECOG 2/3 & $7(4.2)$ & $6(8.5)$ & $1(1.0)$ & \\
\hline \multicolumn{5}{|l|}{ BCLC staging } \\
\hline B & $28(16.7)$ & $8(11.3)$ & 20 (20.6) & \multirow[b]{2}{*}{0.108} \\
\hline $\mathrm{C}$ & $140(83.3)$ & $63(88.7)$ & $77(79.4)$ & \\
\hline \multicolumn{5}{|l|}{ Extrahepatic mets } \\
\hline No & $58(34.5)$ & $19(26.8)$ & $39(40.2)$ & \multirow{2}{*}{0.070} \\
\hline Yes & $110(65.5)$ & $52(73.2)$ & $58(59.8)$ & \\
\hline Macrovascular invasion & & & & \\
\hline No & $84(50.0)$ & $34(47.9)$ & $50(51.5)$ & \\
\hline Yes & $84(50.0)$ & $37(52.1)$ & $47(48.5)$ & 0.639 \\
\hline Child-Pugh score & & & & \\
\hline 5 or 6 & $131(78.0)$ & $51(71.8)$ & $80(82.5)$ & \\
\hline 7 & $23(13.7)$ & $11(15.5)$ & $12(12.4)$ & 0.160 \\
\hline 8 or 9 & $14(8.3)$ & $9(12.7)$ & $5(5.2)$ & \\
\hline ALBI grade & & & & \\
\hline$\leq-2.60$ & $34(20.2)$ & $10(14.1)$ & $24(24.7)$ & \\
\hline$>-2.6$ to $\leq-1.39$ & $119(70.8)$ & $53(74.6)$ & $66(68.0)$ & $0.282^{\wedge}$ \\
\hline$>-1.39$ & $13(7.7)$ & $7(9.9)$ & $6(6.2)$ & $(0.195)$ \\
\hline Unknown & $2(1.2)$ & $1(1.4)$ & $1(1.0)$ & \\
\hline AFP & & & & \\
\hline$<400 \mu \mathrm{g} / \mathrm{L}$ & $91(54.2)$ & $33(46.5)$ & $58(59.8)$ & \\
\hline$\geq 400 \mu \mathrm{g} / \mathrm{L}$ & $76(45.2)$ & $38(53.5)$ & $38(39.2)$ & $0.099 \wedge$ \\
\hline Unknown & $1(0.6)$ & $0(0.0)$ & $1(1.0)$ & (0.074) \\
\hline Etiology & & & & \\
\hline Hepatitis B & $97(57.7)$ & $48(67.6)$ & $49(50.5)$ & \\
\hline Hepatitis C & $15(8.9)$ & $1(1.4)$ & $14(14.4)$ & 0.007 \\
\hline Nonviral & $56(33.3)$ & $22(31.0)$ & $34(35.1)$ & \\
\hline Immunotherapy regimen & & & & \\
\hline IO Monotherapy & $139(82.7)$ & $61(85.9)$ & $78(80.4)$ & \\
\hline IO-IO Combination & $16(9.5)$ & $5(7.0)$ & $11(11.3)$ & 0.600 \\
\hline IO-TKI/mAb combination & $13(7.7)$ & $5(7.0)$ & $8(8.2)$ & \\
\hline
\end{tabular}


Table 1 (continued)

\begin{tabular}{|c|c|c|c|c|}
\hline & \multirow{2}{*}{$\begin{array}{l}\text { Total } \\
(n=168)\end{array}$} & \multicolumn{3}{|l|}{ Frequency (\%) } \\
\hline & & without irAE $(n=71)(42.3)$ & with irAE $(n=97)(57.7)$ & $p$ value \\
\hline \multicolumn{5}{|l|}{ Immunotherapy } \\
\hline Monotherapy & $139(82.7)$ & $61(85.9)$ & 78 (80.4) & \multirow{2}{*}{0.351} \\
\hline Combination & $29(17.3)$ & $10(14.1)$ & $19(19.6)$ & \\
\hline \multicolumn{5}{|c|}{ Immunotherapy (line of therapy) } \\
\hline First line & $114(67.9)$ & $48(67.6)$ & $66(68.0)$ & \multirow{4}{*}{$0.863 \wedge$} \\
\hline Second line & $44(26.2)$ & $18(25.4)$ & $26(26.8)$ & \\
\hline Third line & $9(5.4)$ & $4(5.6)$ & $5(5.2)$ & \\
\hline Fourth line & $1(0.6)$ & $1(1.4)$ & $0(0.0)$ & \\
\hline
\end{tabular}

$p$ value estimated using $x^{2}$ test unless otherwise stated. $p$ value within parenthesis excludes the category "unknown/NA." irAE, immune-related adverse event; SD, standard deviation; IQR, interquartile range. " $p$ value estimated using Mann-Whitney $\mathrm{U}$ test. $\wedge p$ value estimated using Fisher's exact test.

curred more than 2 weeks apart. The study was approved by the centralized institutional review board (CIRB:2018/3046).

\section{Data Processing}

Radiological treatment response was evaluated using the Response Evaluation Criteria in Solid Tumors Version 1.1. Primary endpoints comprised of overall survival (OS) and progression-free survival (PFS), while secondary endpoints comprised of objective response rate (ORR) and disease control rate (DCR). ORR was defined as the proportion of patients who had achieved a best response of complete response (CR) or partial response (PR), while DCR was the proportion of patients who had achieved CR, PR, or stable disease (SD). PFS was calculated as the interval between the date of starting ICI and that of disease progression or death. Patients who were alive but without progression were censored at the time of last follow-up. OS was calculated from the date of starting ICI to the date of death due to all-cause. Patients who are alive at the date of data cutoff were censored at the time of last follow-up. Patients who were lost to follow-up were censored at the time of last known contact.

\section{Statistical Analysis}

Survival curves were estimated by Kaplan-Meier method, and median survival time was reported with $95 \%$ confidence interval (95\% CI). The log-rank test was used to determine if there was a difference in survival curves between groups of patients. Univariable and multivariable analyses were performed using the Cox proportional hazard model. Patient demographics and clinical characteristics associated with survival in the univariable Cox regression model with a significance level of $p<0.1$ and known prognostic factors (ECOG, Child-Pugh, HCC etiology, and AFP levels) were included for model selection. Variable selection was performed using a backward selection strategy using the likelihood ratio test with $p<0.05$ as the criteria for inclusion in the final multivariable model, except for the known prognostic factors. The type of ICI treatment (ICI monotherapy vs. ICI-ICI combination therapy vs. ICI-MAB/TKI therapy) was also added into the final multivariable model to account for difference in treatment effect with differing regimes. Proportionality assumption for using the
Cox regression model was assessed using the Schoenfeld residuals test. Complementary landmark analyses for OS were performed using landmarks of 6 weeks and 12 weeks [21]. $\chi^{2}$ test or Fisher's exact test (where appropriate) was applied to compare ORR and DCR in patients with irAEs and those without. Multivariable logistic regression analyses were used to determine if there was an association between ORR and DCR with the presence of irAEs controlled for the same variables described for the survival analyses. The $\chi^{2}$ test and Student's $t$ test (or the nonparametric tests of Fisher's exact test and Mann-Whitney U test, respectively) were used to assess for differences in demographics between patients with and without irAEs. Multivariable logistic regression analysis was used to determine predictors of irAEs in patients. All $p$ values were based on a two-sided hypothesis, and those of $<0.05$ were considered statistically significant. All analyses were performed using SPSS software (version 25; International Business Machines Corporation) and Stata version 16 (StataCorp. 2019. Stata Statistical Software: Release 16. College Station, TX, USA, StataCorp LLC).

\section{Results}

\section{Patient's Demographics}

One hundred and sixty-eight patients were included in this study. The median follow-up time was 25.1 months (95\% CI: 22.3-35.4 months). The median age was 69 years. $85.7 \%$ of patients were male, and $57.7 \%$ had hepatitis $\mathrm{B}$ infection. The majority of patients were Chinese (67.9\%). 60.7\% had ECOG 0. 78.0\% had Child-Pugh A liver cirrhosis. $50.0 \%$ had macrovascular invasion, and $65.5 \%$ had extrahepatic metastasis. $45.2 \%$ had an AFP of $\geq 400 \mu \mathrm{g} / \mathrm{L}$. $82.7 \%$ of patients received immunotherapy monotherapy, while $17.3 \%$ received immunotherapy in combination (Table 1). A more detailed breakdown of 
Table 2. Frequency of irAE (all patients)

\begin{tabular}{llllll}
\hline & \multicolumn{2}{l}{ Patients with irAE, $n(\%)$} & Patients with systemic & $\begin{array}{l}\text { Patients with treatment } \\
\text { discontinuation, } n(\%)\end{array}$ \\
\cline { 2 - 4 } & Total & Grades 1-2 & Grade $\geq 3$ & & $28(16.7)$ \\
\hline Any irAE & $97(57.7)$ & $90(53.6)$ & $24(14.3)$ & $27(16.1)$ & $5(3.0)$ \\
Dermatological irAE & $79(47.0)$ & $77(45.8)$ & $2(1.2)$ & $8(4.8)$ & $11(7.1)$ \\
Hepatobiliary irAE & $24(14.3)$ & $12(7.1)$ & $12(7.1)$ & $11(6.5)$ & $2(1.2)$ \\
Endocrine irAE & $16(9.5)$ & $14(8.3)$ & $2(1.2)$ & $1(0.6)$ & $4(2.4)$ \\
Gastrointestinal irAE & $15(8.9)$ & $10(6.0)$ & $5(3.0)$ & $4(2.4)$ & $8(4.8)$ \\
Pneumonitis irAE & $9(5.4)$ & $5(3.0)$ & $4(2.4)$ & $8(4.8)$ & - \\
Musculoskeletal irAE & $3(1.8)$ & $3(1.8)$ & - & $1(0.6)$ & $1(0.6)$ \\
Hematology irAE & $2(1.2)$ & $1(0.6)$ & $1(0.6)$ & $1(0.6)$ & $1(0.6)$ \\
Neurology irAE & $1(0.6)$ & $1(0.6)$ & - & - & - \\
Renal irAE & $1(0.6)$ & - & $1(0.6)$ & $1(0.6)$ & $2(1.2)$ \\
Others irAE & $11(6.5)$ & $10(6.0)$ & $1(0.6)$ & & \\
1 irAE & $61(36.3)$ & & & & \\
2 irAE & $22(13.1)$ & & & & \\
$\geq 3$ irAE & $14(8.3)$ & & & & \\
\hline
\end{tabular}

irAE, immune-related adverse event.

treatment regime can be found in online supplementary Table 1; for all online supplementary material, see www. karger.com/doi/10.1159/000518619.

Older age, male gender, ECOG 0 (vs. 2/3), and hepatitis $\mathrm{C}$ etiology were more likely to experience irAE on the univariable analysis (Table 1). Older age, male gender, ECOG 0 (vs. 2/3), and hepatitis $C$ remained significant on the multivariable analysis (online suppl. Table 2). Patients who received combination therapy were more likely to experience grade $\geq 3 \mathrm{irAE}$ than patients who received ICI monotherapy ( 31.0 vs. $10.8 \%, p=0.009$ ) (online suppl. Table 3).

\section{Incidence and Time to Onset of irAEs}

Ninety-seven patients (57.7\%) experienced all-grade irAEs, while 24 (14.3\%) experienced grade $\geq 3$ irAEs. Two patients (1.2\%) experienced treatment-related death. The most common all-grade irAEs were dermatological (79, $47 \%)$, hepatobiliary $(24,14.3 \%)$, and endocrine irAEs (16, $9.5 \%)$. The most common grade $\geq 3$ irAEs were hepatobiliary $(12,7.1 \%)$, gastrointestinal $(5,3.0 \%)$, and pneumonitis $(4,2.4 \%)$. Sixty-one (36.3\%) of patients experienced 1 irAE, 22 (13.1\%) experienced 2 irAEs, and 14 (8.3\%) experienced $\geq 3$ irAEs. Of the patients who experienced $\geq 2$ irAEs, 15 had concurrent irAEs, while 21 had sequential irAEs (Table 2). The frequency of irAEs by treatment regimens (ICI monotherapy, ICI-ICI combination, and ICI-MAB/TKI combination) can be found in online supplementary Table 4.

Impact of irAE on Efficacy of ICI in HCC
Median time to onset was shortest for hepatobiliary irAEs (3.9 weeks) and longest for pneumonitis (43.3 weeks). This is further described in online supplementary Figure 1.

\section{Association of irAE and Response}

Patients with all-grade irAEs had a significantly higher ORR (27.8\% vs. $11.3 \%, p=0.009)$ and DCR (67.0\% vs. $28.2 \%, p=<0.001)$ as compared with patients without irAEs. The ORR for patients with grade $\geq 3$ irAE versus grades $1-2$ irAE versus no irAE was $50.0 \%, 20.5 \%$, and $11.3 \%$, respectively $(p<0.001)$. The DCR for patients with grade $\geq 3$ irAE versus grades $1-2$ irAE versus no irAE was $87.5 \%, 60.3 \%$, and $28.2 \%$, respectively $(p<0.001)$.

In the multivariable analysis, patients with grade $\geq 3$ irAE demonstrated improved ORR (adjusted odds ratio $[\mathrm{aOR}], 4.89, p=0.002)$ versus those with no irAEs. Patients with grade $\geq 3$ irAE and grades $1-2$ irAE demonstrated improved DCR (aOR, 13.8, $p<0.001$, and 3.42, $p=0.001$, respectively) versus those with no irAEs. The presence of grade $\geq 3$ irAE, dermatological, endocrine, and $\geq 2$ irAEs were associated with ORR, while the presence of any grade irAE, grade $\geq 3$ irAE, dermatological, endocrine, hepatobiliary, gastrointestinal, one irAE, and $\geq 2$ irAEs were associated with DCR (Table 3 and online suppl. Table 5). 
Table 3. Association of irAE and ORR/DCR

\begin{tabular}{|c|c|c|c|c|}
\hline & \multicolumn{2}{|c|}{ Multivariable (endpoint of ORR)* } & \multicolumn{2}{|c|}{ Multivariable (endpoint of DCR)** } \\
\hline & adjusted OR (95\% Cl) & $p$ value & adjusted OR (95\% Cl) & $p$ value \\
\hline Any irAE & $2.39(0.95,5.98)$ & 0.063 & $4.47(2.18,9.19)$ & $<0.001$ \\
\hline Any grade $\geq 3$ irAE & $4.89(1.76,13.61)$ & 0.002 & $7.08(1.96,25.64)$ & 0.003 \\
\hline Grades $1-2$ irAE versus no irAE & $1.61(0.60,4.34)$ & 0.346 & $3.42(1.61,7.25)$ & 0.001 \\
\hline Grade $\geq 3$ irAE versus no irAE & $6.45(1.97,21.1)$ & 0.002 & $13.79(3.54,53.70)$ & $<0.001$ \\
\hline Dermatological irAE & $2.43(1.03,5.71)$ & 0.042 & $3.46(1.71,6.98)$ & $<0.001$ \\
\hline Pneumonitis irAE & $2.41(0.52,11.26)$ & 0.264 & $7.00(0.83,59.35)$ & 0.074 \\
\hline Endocrine irAE & $4.39(1.43,13.49)$ & 0.010 & $13.66(1.72,108.59)$ & 0.013 \\
\hline Hepatobiliary irAE & $1.34(0.47,3.81)$ & 0.583 & $4.57(1.42,14.74)$ & 0.011 \\
\hline Gastrointestinal irAE & $1.85(0.51,6.72)$ & 0.352 & $5.81(1.20,28.14)$ & 0.029 \\
\hline 1 irAE versus no irAE & $1.97(0.72,5.36)$ & 0.185 & $3.00(1.40,6.46)$ & 0.005 \\
\hline$\geq 2$ irAE versus no irAE & $3.28(1.10,9.81)$ & 0.033 & $13.60(4.25,43.52)$ & $<0.001$ \\
\hline
\end{tabular}

$p$ value calculated using Wald test. * Covariables included in the multivariate analysis included those that showed a significant association in univariable analysis such as etiology (hepatitis B vs. hepatitis $C$ vs. nonviral), as well as predetermined ones such as AFP $(<400 \mu \mathrm{g} / \mathrm{L}$ vs. $\geq 400 \mu \mathrm{g} / \mathrm{L}$ vs. unknown) and ICl treatment (ICI monotherapy vs. ICl-ICl combination vs. ICI-MAB/TKI combination). ${ }^{* *}$ Covariables included in the multivariate analysis included those that showed a significant association in univariable analysis such as Child-Pugh score (5/6 vs. 7 vs. 8/9), as well as predetermined ones such as etiology (hepatitis B vs. hepatitis C vs. nonviral), AFP $(<400 \mu \mathrm{g} / \mathrm{L}$ vs. $\geq 400 \mu \mathrm{g} / \mathrm{L}$ vs. unknown), and ICl treatment (ICl monotherapy vs. ICI-ICl combination vs. ICl-MAB/TKI combination). NA, not applicable; NE, not estimable; $\mathrm{Cl}$, confidence interval; OR, odds ratio; ORR, objective response rate; DCR, disease control rate; irAE, immune-related adverse event; ICl, immune checkpoint inhibitor; TKI, tyrosine kinase inhibitor.

\section{Association of irAE and Survival}

Patients with all-grade irAEs had a longer median PFS as compared with patients without irAEs (5.5 mths [95\% CI: 3.4-8.2] vs. $1.3 \mathrm{mths}$ [95\% CI: $1.1-1.6]$, hazard ratio (HR) 0.43 [95\% CI: $0.31-0.61], p<0.001$ ). The median PFS for patients with grade $\geq 3$ irAE versus grades $1-2$ irAE versus no irAE was $8.5 \mathrm{mths}$ (95\% CI: $7.8-19.1)$ versus $3.6 \mathrm{mths}(95 \% \mathrm{CI}: 1.8-6.8)$ versus 1.3 mths (95\% CI: 1.1-1.6) $(p<0.001)$. The median PFS for patients with $\geq 2$ irAE versus one versus no irAE was $10.1 \mathrm{mths}$ (95\% CI: 7.7-19.1) versus $2.8 \mathrm{mths}$ (95\% CI: $1.7-5.5)$ versus $1.3 \mathrm{mths}$ (95\% CI: $1.1-1.6)(p<0.001)$. The presence of dermatological, pneumonitis, and endocrine irAEs were also associated with a significantly longer PFS on univariable analysis (Fig. 1; Table 4, online suppl. Table 6).

In the multivariable analysis, patients with grade $\geq 3$ ir AE and grades 1-2 irAE demonstrated improved PFS (adjusted HRs [aHRs], 0.38 and 0.52 , respectively, both $p$ $<0.001$ ) versus those with no irAEs, while patients with $\geq 2$ irAEs and 1 irAE demonstrated improved PFS (aHRs, $0.36, p<0.001$, and $0.57, p=0.005$, respectively) versus those with no irAEs. In addition, the presence of all-grade ir $\mathrm{AE}$, grade $\geq 3$ irAE, dermatological ir $\mathrm{AE}$, and endocrine irAE were associated with a significantly longer median
PFS. Covariables included in the analysis were etiology of HCC, Child-Pugh score, AFP, ECOG, and type of ICI treatment (Table 4).

Patients with all-grade irAEs had a significant longer median OS as compared with patients without irAEs (16.2 mths [95\% CI: 13.9-20.7] vs. $4.6 \mathrm{mths}$ [95\% CI: $3.2-$ 5.7], HR 0.45 [95\% CI: 0.31-0.66], $p<0.001$ ). The median OS for grade $\geq 3$ irAE versus grades $1-2$ irAE versus no irAE was 26.9 mths (95\% CI: 15.8-not estimable [NE]) versus 14.0 mths (95\% CI: 9.7-19.8) versus $4.6 \mathrm{mths}$ (95\% CI: 3.2-5.7) $(p<0.001)$. The median OS for patients with $\geq 2$ irAE versus one versus no irAE was $20.7 \mathrm{mths}$ ( $95 \%$ CI: $15.8-36.1$ ) versus 13.9 mths (95\% CI: 7.3-18.5) versus 4.6 mths (95\% CI: 3.2-5.7) $(p<0.001)$. The presence of dermatological and pneumonitis irAEs were also associated with a significantly longer OS on univariable analysis (Fig. 1; Table 5, online suppl. Table 7).

In the multivariable analysis, patients with grade $\geq 3$ irAE and grades 1-2 irAE demonstrated improved OS (aHRs, $0.28, p<0.001$, and $0.57, p=0.010$, respectively) versus those with no irAEs, while patients with $\geq 2$ irAEs and 1 irAE demonstrated improved OS (aHRs, $0.35, p<$ 0.001 , and $0.59, p=0.020$, respectively) versus those with no irAEs. In addition, the presence of all-grade irAE, grade $\geq 3$ irAE, and dermatological irAEs were associated
14

Liver Cancer 2022;11:9-21 DOI: $10.1159 / 000518619$
Ng/Tan/Tan/Tay/Lee/Ang/Wong/Choo/ Tai/Lee 


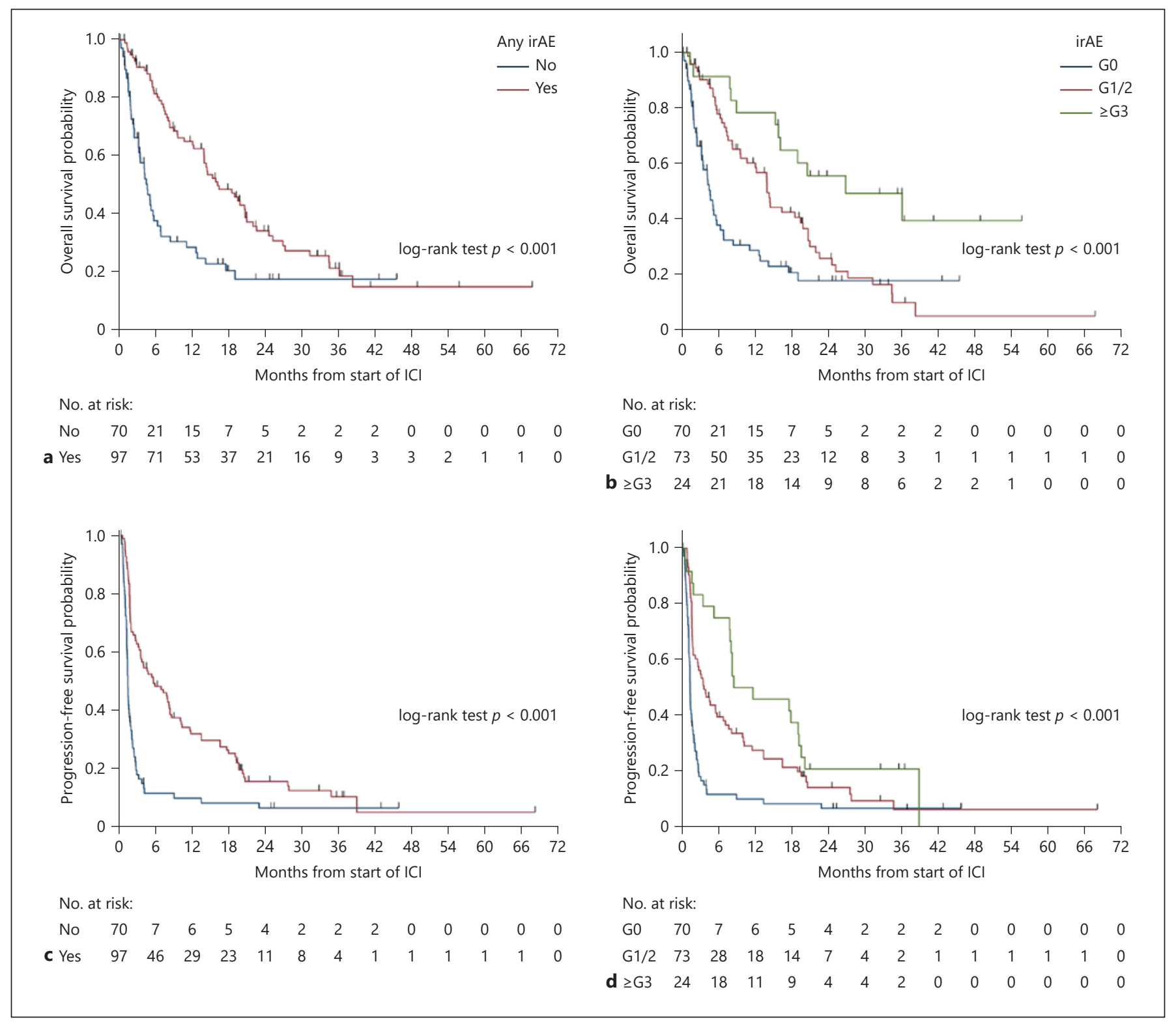

Fig. 1. a OS (any irAE vs. no irAE); (b): OS (grade $\geq 3$ vs. grades 1-2 vs. no irAE); (c): PFS (any irAE vs. no irAE); (d): PFS (grade $\geq 3$ vs. grades 1-2 vs. no irAE). irAE, immune-related adverse event; ICI, immune checkpoint inhibitor; OS, overall survival; PFS, progression-free survival.

with a significantly longer median OS. Covariables included in the analysis were etiology of HCC, Child-Pugh score, AFP, ECOG, and type of ICI treatment (Table 5).

A complementary landmark analysis was performed at the 6-week and 12-week landmarks. The presence of grade $\geq 3$ irAE was associated with a significant longer OS on the univariable and multivariable analysis at the 6-week landmark (HR 0.36, 95\% CI: 0.14-0.88, $p=0.026$ and aHR 0.34, 95\% CI: 0.13-0.90, $p=0.030$ ) and 12 -week landmark (HR 0.31, 95\% CI: 0.13-0.78, $p=0.012$ and aHR $0.28,95 \%$ CI: $0.11-0.75, p=0.011)$. The presence of $\geq 2$ irAEs was associated with a trend toward longer OS at the 6-week landmark (HR 0.41, 95\% CI: 0.16-1.03, $p=0.057$ and aHR 0.37, 95\% CI: 0.13-1.07, $p=0.067$ ) (online suppl. Table 8, online suppl. Fig. 2). Sensitivity analysis restricting to patients treated with ICI monotherapy or ICIICI combination therapy demonstrated results consistent with the main analysis (online suppl. Tables 9, 10). 
Table 4. Association of irAE and PFS

\begin{tabular}{|c|c|c|c|c|}
\hline \multirow[t]{2}{*}{ PFS (events/patients = 146/167) } & \multicolumn{2}{|l|}{ Univariable } & \multicolumn{2}{|l|}{ Multivariable } \\
\hline & $\mathrm{HR}(95 \% \mathrm{Cl})$ & $p$ value & $\operatorname{aHR}(95 \% \mathrm{Cl})$ & $p$ value \\
\hline Any irAE & $0.43(0.31,0.61)^{\#}$ & $<0.001$ & $0.48(0.33,0.69)^{\#}$ & $<0.001$ \\
\hline Any grade $\geq 3$ irAE & $0.51(0.32,0.82)^{\#}$ & 0.005 & $0.57(0.34,0.95)^{\#}$ & 0.030 \\
\hline Grades $1-2$ irAE versus no irAE & $0.48(0.34,0.69)^{\#}$ & $<0.001$ & $0.52(0.35,0.76)^{\#}$ & $<0.001$ \\
\hline Grade $\geq 3$ irAE versus no irAE & $0.32(0.19,0.54)^{\#}$ & $<0.001$ & $0.38(0.22,0.66)^{\#}$ & $<0.001$ \\
\hline Dermatological irAE & $0.47(0.33,0.65)^{\#}$ & $<0.001$ & $0.50(0.35,0.71)^{\#}$ & $<0.001$ \\
\hline Pneumonitis irAE & $0.43(0.20,0.92)^{\#}$ & 0.030 & $0.46(0.21,1.01)^{\#}$ & 0.052 \\
\hline Endocrine irAE & $0.41(0.23,0.75)$ & 0.004 & $0.48(0.26,0.88)$ & 0.019 \\
\hline Hepatobiliary irAE & $0.68(0.43,1.07)^{\#}$ & 0.093 & $0.74(0.44,1.24)$ & 0.252 \\
\hline Gastrointestinal irAE & $0.71(0.41,1.23)^{\#}$ & 0.224 & $0.92(0.50,1.70)$ & 0.785 \\
\hline 1 irAE versus no irAE & $0.54(0.38,0.79)^{\#}$ & 0.001 & $0.57(0.38,0.84)^{\#}$ & 0.005 \\
\hline$\geq 2$ irAE versus no irAE & $0.31(0.20,0.49)^{\#}$ & $<0.001$ & $0.36(0.22,0.59)^{\#}$ & $<0.001$ \\
\hline
\end{tabular}

Covariables included in the multivariate analysis included those that showed a significant association in univariable analysis such as etiology (hepatitis B vs. hepatitis C vs. nonviral), Child-Pugh score (5/6 vs. 7 vs. 8/9), and $\operatorname{AFP}(<400 \mu \mathrm{g} / \mathrm{L}$ vs. $\geq 400 \mu \mathrm{g} / \mathrm{L}$ vs. unknown), as well as predetermined ones such as ECOG (0 vs. 1 vs. $2 / 3)$ and ICI treatment (ICl monotherapy vs. ICl-ICl combination vs. ICI-MAB/TKI combination). $p$ value calculated using Wald test. NA, not applicable; NE, not estimable; $\mathrm{Cl}$, confidence interval; PFS, progression-free survival; irAE, immunerelated adverse event; ICl, immune checkpoint inhibitor; TKI, tyrosine kinase inhibitor; aHR, adjusted HR; HR, hazard ratio. " Proportional hazard assumption violated.

Table 5. Association of irAE and OS

\begin{tabular}{|c|c|c|c|c|}
\hline \multirow[t]{2}{*}{ OS (events/patients $=111 / 167$ ) } & \multicolumn{2}{|l|}{ Univariable } & \multicolumn{2}{|l|}{ Multivariable } \\
\hline & $\mathrm{HR}(95 \% \mathrm{Cl})$ & $p$ value & adjusted HR (95\% Cl) & $p$ value \\
\hline Any irAE & $0.45(0.31,0.66)^{\#}$ & $<0.001$ & $0.49(0.32,0.74)^{\#}$ & $<0.001$ \\
\hline Any grade $\geq 3$ irAE & $0.36(0.20,0.67)$ & 0.001 & $0.40(0.21,0.76)$ & 0.005 \\
\hline Grades $1-2$ irAE versus no irAE & $0.54(0.36,0.81)^{\#}$ & 0.003 & $0.57(0.37,0.87)^{\#}$ & 0.010 \\
\hline Grade $\geq 3$ irAE versus no irAE & $0.25(0.13,0.48)$ & $<0.001$ & $0.28(0.14,0.56)$ & $<0.001$ \\
\hline Dermatological irAE & $0.45(0.31,0.66)^{\#}$ & $<0.001$ & $0.50(0.33,0.75)^{\#}$ & $<0.001$ \\
\hline Pneumonitis irAE & $0.40(0.16,0.97)^{\#}$ & 0.044 & $0.43(0.17,1.07)^{\#}$ & 0.069 \\
\hline Endocrine irAE & $0.55(0.29,1.06)$ & 0.075 & $0.69(0.35,1.36)$ & 0.288 \\
\hline Hepatobiliary irAE & $0.58(0.34,1.01)$ & 0.052 & $0.58(0.31,1.07)$ & 0.082 \\
\hline Gastrointestinal irAE & $0.52(0.25,1.07)$ & 0.075 & $0.70(0.31,1.58)$ & 0.387 \\
\hline 1 irAE versus no irAE & $0.59(0.39,0.90)^{\#}$ & 0.013 & $0.59(0.37,0.92)^{\#}$ & 0.020 \\
\hline$\geq 2$ irAE versus no irAE & $0.30(0.18,0.51)^{\#}$ & $<0.001$ & $0.35(0.20,0.62)^{\#}$ & $<0.001$ \\
\hline
\end{tabular}

Covariables included in the multivariate analysis included those that showed a significant association in univariable analysis such as ECOG (0 vs. 1 vs. 2/3), etiology (hepatitis B vs. hepatitis C vs. nonviral), Child-Pugh score (5/6 vs. 7 vs. $8 / 9)$, and AFP ( $<400 \mu \mathrm{g} / \mathrm{L}$ vs. $\geq 400 \mu \mathrm{g} / \mathrm{L}$ vs. unknown), as well as predetermined ones such as ICI treatment (ICl monotherapy vs. ICl-ICl combination vs. ICl-MAB/TKI combination). $p$ value calculated using Wald test. NA, not applicable; $\mathrm{NE}$, not estimable; $\mathrm{Cl}$, confidence interval; irAE, immune-related adverse event; $\mathrm{ICl}$, immune checkpoint inhibitor; TKI, tyrosine kinase inhibitor; OS, overall survival; aHR, adjusted HR; HR, hazard ratio. \# Proportional hazard assumption violated.

Pattern of Systemic Steroid Usage to Treat Patients with irAE and Impact on ICI Efficacy

Twenty-seven (16.1\%) of patients received systemic steroids for treatment of irAEs. Ten (37.0\%), 11 (40.7\%), and $6(22.2 \%)$ started at a dose of oral prednisolone equivalent of $\leq 0.5 \mathrm{mg} / \mathrm{kg},>0.5 \mathrm{mg} / \mathrm{kg}$, and intravenous systemic steroids, respectively. Two (7.4\%) received adjunctive immunosuppressants (1 patient received cyclospo-
16

Liver Cancer 2022;11:9-21

DOI: $10.1159 / 000518619$
Ng/Tan/Tan/Tay/Lee/Ang/Wong/Choo/ Tai/Lee 
rine, while the other received anti-tumor necrosis factor monoclonal antibodies). The median duration of systemic steroid therapy was 59 days with an interquartile range of 21-94. Five (18.5\%) received multiple courses of systemic steroids.

In patients with irAE, those who receive systemic steroids have a trend toward longer PFS as compared to those who did not receive systemic steroids $(9.9 \mathrm{mths}$ [95\% CI: 7.8-17.8] vs. 3.4 mths [95\% CI: 1.8-5.5], HR 0.75 [95\% CI: $0.46-1.21], p=0.238)$. The same trend was observed for OS (20.7 mths [95\% CI: 15.3-NE] vs. $14.3 \mathrm{mths}$ [95\% CI: 9.6-21.0], HR 0.59 [95\% CI: 0.33-1.04], $p=$ $0.068)$. The starting dose of systemic steroids did not have an impact on PFS or OS. However, the duration of systemic steroids had an impact. Patients with irAE receiving systemic steroids for $\geq 60$ days had a longer PFS and OS, respectively, as compared to those who received $<60$ days of treatment (online suppl. Tables 6,7).

\section{Discussion}

Our study suggests that the presence of all-grade irAEs may be a potential prognostic biomarker in patients with aHCC treated with ICI. Patients who experienced multisystem irAEs ( 2 or more systems) and more severe irAEs had a significantly higher ORR, DCR, and longer PFS and OS as compared to the patients with no irAEs. Among patients who experienced irAEs, the usage of systemic corticosteroids to manage irAEs was not associated with a detrimental effect on PFS and OS.

Presence of irAE as a Potential Predictive Biomarker for ICI Efficacy in Patients with aHCC

Patients who experienced irAEs in our study had superior OS (16.2 mths vs. $4.6 \mathrm{mths}$ ), PFS (5.5 mths vs. 1.3 mths), ORR (27.8\% vs. $11.3 \%)$, and DCR (67.0\% vs. $28.2 \%)$. This adds to the growing body of evidence that has demonstrated the association of irAE and efficacy of programmed cell death protein-1/programmed death ligand-1 inhibitors in patients with NSCLC [22, 23], melanoma $[9,24]$, urothelial carcinoma [10], head and neck cancer [25], and gastrointestinal cancers [14]. There are also studies performed in metastatic melanoma demonstrating the association of irAEs and efficacy of cytotoxic T lymphocyte antigen-4 (CTLA-4) inhibitors [26, 27]. No studies have described this association in patients receiving combination immunotherapy, and our study suggests that the association is likely to be also present for patients who are receiving combination immunotherapy or im-

Impact of irAE on Efficacy of ICI in HCC munotherapy in combination with TKI or vascular endothelial growth factor inhibitor.

Mechanistically, patients who experience a higher grade irAE should have a higher T-cell activity and, hence, experience better antitumor outcomes than patients who experience a lower grade irAE [28]. Our study seems to support this hypothesis as patients who experienced a grade $\geq 3$ irAE had significantly longer OS, PFS, and higher ORR and DCR. Contrary to our findings, existing studies in melanoma and gastrointestinal cancers have not demonstrated a difference in ICI efficacy between patients with higher grade irAE and lower grade irAE $[14,24]$. In the same way, one may infer that patients who experience irAEs at multiple sites would have greater T-cell activity and hence experience better antitumor outcomes. Our study showed that patients with more sites of irAEs had a significant longer PFS as compared to those with fewer sites of involvement or no irAE ( $\geq 2$ irAEs: 10.1 mths vs. 1 irAE: 2.8 mths vs. 0 irAE: 1.3 $\mathrm{mths}, p<0.001)$. The same association was seen for OS ( $\geq 2$ irAEs: 20.7 mths vs. 1 irAE: 13.9 mths vs. 0 irAE: 4.6 mths, $p<0.001$ ). Shankar et al. [29] described similar results in a multicenter cohort of patients with NSCLC. Patients with irAEs at more sites had a significantly longer PFS and OS.

The specific site of irAE may be associated with efficacy of ICI, and this may vary in different malignancies. In patients with metastatic melanoma, it has been demonstrated that the presence of dermatological ir AE (especially vitiligo) was associated with significantly longer PFS and OS [30]. In contrast, Ricciuti et al. [23] demonstrated in patients with NSCLC that pneumonitis, gastrointestinal, and endocrine irAEs but not dermatologic or hepatobiliary irAE were associated with a significantly longer OS. The available data seem to suggest that a potential molecular mimicry between malignant and normal cells may explain the association between certain sites of irAE and ICI efficacy (e.g., shared antigen between melanocytes and the metastatic melanoma cells). One would hypothesize that in patients with HCC, the presence of hepatobiliary irAE would be associated with increased ICI efficacy. In our study, there was only a trend toward a longer PFS and OS in the multivariable analysis in patients experiencing hepatobiliary irAE. On the other hand, patients with dermatological irAE had a significantly longer OS, while patients with dermatological and endocrine irAE had a significantly longer PFS. Clearly, more work is required in this field to ascertain if site of irAE matters and the underlying mechanism. 


\section{Role of Steroids}

We found that patients who received systemic corticosteroids for treatment of irAEs had a trend toward longer OS and PFS as compared to patients with irAEs who did not receive systemic corticosteroids. This is likely because most of the patients who received systemic corticosteroids experienced grade $\geq 3$ irAEs and the presence of grade $\geq 3$ irAEs was associated with a significantly longer OS and PFS than patients without grade $\geq 3$ irAEs. There were initial concerns regarding poorer outcomes with the usage of corticosteroids in patients receiving ICI [31], and patients receiving corticosteroids at baseline were excluded from clinical trials studying the use of ICI in HCC [4, 7]. However, a meta-analysis and systematic review (including studies consisted mainly of patients with lung cancer and melanoma; no patients with HCC were included) demonstrated that the negative association of systemic corticosteroids and outcomes may be related to the indication of corticosteroids. While the usage of systemic corticosteroids for cancer-related symptoms was correlated with a significantly shorter OS and PFS, the usage of systemic corticosteroids for treatment of irAEs was not associated with a shorter OS [17]. Pinato et al. [32] described in a cohort of 304 patients with HCC that the usage of systemic corticosteroids at baseline or concurrently with ICI was not associated with worse OS, PFS, or ORR. However, usage of systemic corticosteroids for cancer-related symptoms was associated with a shorter PFS and lower ORR. Putting these together, our data add to the body of evidence that the usage of systemic corticosteroids to treat irAEs is safe and should be done promptly. Interestingly, a longer duration of systemic corticosteroid usage was associated with a longer PFS and OS. This suggests that the long-term usage of systemic corticosteroids to manage irAEs may not be detrimental to the efficacy of ICI-based regimes.

\section{Incidence, Time to Onset, and Predictors of irAE}

In our study, we found that $57.7 \%$ of patients experienced irAEs of any grades, $14.3 \%$ experienced grade $\geq 3$ irAEs, and $1.2 \%$ experienced treatment-related death. There was a higher incidence of irAEs seen with combination immunotherapy or immunotherapy combined with TKI or vascular endothelial growth factor inhibitor than monotherapy immunotherapy. This is similar to what has been reported in the phase 2 and phase 3 studies leading to the approval of various immunotherapy regimes [4, $6-8,33,34]$.

We found that older, male patients, those with better performance status (PS) and those with hepatitis C, had a higher incidence of all-grade irAEs. While it is a welldocumented fact that chemotherapy toxicity increases with age [35], this relationship is less clear in patients treated with ICI. van Holstein et al. [36] described in a review that the majority of studies (clinical trials or retrospective studies) did not suggest a higher incidence of irAEs in older patients treated with ICI. However, there may be a trend toward early treatment discontinuation and more patients requiring treatment with immunosuppressants. A large retrospective study including $527 \mathrm{pa}-$ tients with NSCLC treated with ICI outside of a clinical trial demonstrated no difference in incidence of irAEs (any grades) or systemic corticosteroid usage, but there was a trend toward early treatment interruption at 6 weeks for patients $\geq 75$ years old. Valpione et al. [37] reported that female patients were more likely to experience irAEs in a study of patients with melanoma treated with ipilimumab. This is contrary to our findings. The differences in the underlying malignancy (melanoma vs. aHCC) and type of ICI used (predominance of programmed cell death protein-1/PD-L1 inhibitors vs. cytotoxic $\mathrm{T}$ lymphocyte antigen- 4 inhibitors) may preclude direct comparison. Nevertheless, large meta-analysis suggested that males may benefit more from ICI. The effects of gender on the immune system are complex, and how this influences the efficacy and incidence of irAEs remains an area that requires active research [38]. In contrast to the known association between poor PS and chemotherapy toxicities, in our cohort, patients with better PS had a higher incidence of irAEs [35]. However, it could be possible that patients with a better PS have a more robust immune system and could hence mount an immune response that leads to development of irAEs [39]. Another possible explanation could be that patients with better PS have longer PFS and OS [40, 41] and, hence, have a longer duration of ICI treatment. This could account for the higher incidence of irAEs. The same line of reasoning could explain why patients with HCC secondary to hepatitis $\mathrm{C}$ (a known positive prognostic factor) would have a higher incidence of irAEs.

\section{Strengths and Limitations}

This study has several limitations. First, it is a singlecenter study. Second, the study is retrospective in nature and information bias cannot be excluded. In addition, the predominance of hepatitis B-associated HCC in this study (a known negative prognostic factor) precludes generalization of this study data to non-hepatitis B-associated HCC. Also, the definition of whether a patient experienced irAE is at the investigator's discretion. How-
18

Liver Cancer 2022;11:9-21 DOI: $10.1159 / 000518619$
Ng/Tan/Tan/Tay/Lee/Ang/Wong/Choo/ Tai/Lee 
ever, all patients' clinical charts are reviewed by at least two oncologists when defining an irAE event.

The strength of this study includes it being the largest study describing such an association in patients with aHCC. Previous studies described 32 and 101 patients, respectively $[14,42]$. The study including 101 patients did not include OS as an outcome. In addition, the long median follow-up of 25.1 months allows analysis of longterm survival outcomes. Finally, the use of landmark analysis allowed us to account for immortal bias which was not included in the previous 2 studies.

\section{Conclusion}

Our study suggests that the presence of irAEs may be a potential prognostic biomarker in patients with aHCC treated with ICI. Patients with more severe irAEs and multisystem involvement have better prognosis. The prompt use of systemic corticosteroids to treat patients with irAEs is key to ensure the best long-term outcomes for these patients.

\section{Acknowledgments}

We thank the patients and their families.

\section{Statement of Ethics}

The institutional review board of SingHealth granted ethical approval for this study (2018/3046). All patients have given their written informed consent.

\section{Conflict of Interest Statement}

S.P.C. has received research funding and speaking fees from Bristol Myers Squibb (BMS), speaking fees from Lilly, and research funding from Sirtex, and has participated on advisory boards for BMS, Sirtex, Lilly, Novartis, Eisai, Bayer, and Celgene. D.W.-M.T. has received research funding for BMS and Sirtex, and honorarium from Bayer and has participated on advisory boards for Eisai, Bayer, and Ipsen. J.J.X.L. has received research funding from Bayer and honorarium from BMS and Ipsen, and has participated on advisory boards for Bayer and Ipsen.

\section{Funding Sources}

This study was not funded.

\section{Author Contributions}

K.Y.Y.N., L.W.J.W., A.J.S.A., S.P.C., and J.J.X.L. conceived and designed the study. S.P.C., D.W.-M.T., and J.J.X.L. provided study material or patients. K.Y.Y.N., L.W.J.W., A.J.S.A., A.W.X.L., D.S.H.T., and J.J.E.T. collected the data. All authors analyzed, interpreted the data, and were involved in the writing, review, and approval of the manuscript.

\section{Author Information}

Dr. Kennedy Yao Yi Ng is Senior Resident, Division of Medical Oncology, National Cancer Centre Singapore, Singapore. Dr. Sze Huey Tan is Principal Biostatistician, Division of Clinical Trials and Epidemiological Sciences, National Cancer Centre Singapore, Singapore. Mr. Jack Jie En Tan is Medical Student, Yong Loo Lin School of Medicine, National University of Singapore. Ms Desiree Shu Hui Tay is Medical Student, Yong Loo Lin School of Medicine, National University of Singapore. Ms. Ailica Wan Xin Lee is Medical Student, Yong Loo Lin School of Medicine, National University of Singapore. Dr. Andrea Jing Shi Ang is House Officer, Division of Internal Medicine, Singapore General Hospital, Singapore. Dr. Lawrence Wen Jun Wong is House Officer, Division of Paediatrics, KK Women's and Children's Hospital, Singapore. Dr. Su Pin Choo is Visiting Consultant, Division of Medical Oncology, National Cancer Centre Singapore, Singapore. Dr. David WaiMeng Tai is Senior Consultant, Division of Medical Oncology, National Cancer Centre Singapore, Singapore. Dr. Joycelyn Jie Xin Lee is Consultant, Division of Medical Oncology, National Cancer Centre Singapore, Singapore.

\section{Data Availability Statement}

Data are available on reasonable request to the corresponding author.

References

Liver Cancer 2022;11:9-2

DOI: $10.1159 / 000518619$
1 Torre LA, Bray F, Siegel RL, Ferlay J, LortetTieulent J, Jemal A. Global cancer statistics, 2012. CA Cancer J Clin. 2015;65(2):87-108.

2 Llovet JM, Ricci S, Mazzaferro V, Hilgard P, Gane E, Blanc JF, et al. Sorafenib in advanced hepatocellular carcinoma. N Engl J Med. 2008;359(4):378-90.

3 Kudo M, Finn RS, Qin S, Han KH, Ikeda K, Piscaglia F, et al. Lenvatinib versus sorafenib in first-line treatment of patients with unresectable hepatocellular carcinoma: a randomised phase 3 non-inferiority trial. Lancet. 2018;391(10126):1163-73.

4 Finn RS, Qin S, Ikeda M, Galle PR, Ducreux M, Kim TY, et al. Atezolizumab plus bevacizumab in unresectable hepatocellular carcinoma. N Engl J Med. 2020;382(20):1894-905.

5 Postow MA, Sidlow R, Hellmann MD. Immune-related adverse events associated with immune checkpoint blockade. N Engl J Med. 2018;378(2):158-68. 
6 Yau T, Park JW, Finn RS, Cheng A-L, Mathurin P, Edeline J, et al. LBA38_PRCheckMate 459: a randomized, multi-center phase III study of nivolumab (NIVO) vs sorafenib (SOR) as first-line (1L) treatment in patients (pts) with advanced hepatocellular carcinoma (aHCC). Ann Oncol. 2019; 30(Supplement_5):v851-934.

7 Finn RS, Ryoo BY, Merle P, Kudo M, Bouattour M, Lim HY, et al. Pembrolizumab as second-line therapy in patients with advanced hepatocellular carcinoma in KEYNOTE-240: a randomized, double-blind, phase III trial. J Clin Oncol. 2020;38(3):193-202.

8 Yau T, Kang YK, Kim TY, El-Khoueiry AB, Santoro A, Sangro B, et al. Efficacy and Safety of Nivolumab Plus Ipilimumab in Patients With Advanced Hepatocellular Carcinoma Previously Treated With Sorafenib: the CheckMate 040 Randomized Clinical Trial. JAMA Oncol. 2020;6(11):e204564.

9 Indini A, Di Guardo L, Cimminiello C, Prisciandaro M, Randon G, De Braud F, et al. Immune-related adverse events correlate with improved survival in patients undergoing anti-PD1 immunotherapy for metastatic melanoma. J Cancer Res Clin Oncol. 2019;145(2): 511-21.

10 Maher VE, Fernandes LL, Weinstock C, Tang S, Agarwal S, Brave M, et al. Analysis of the association between adverse events and outcome in patients receiving a programmed death protein 1 or programmed death ligand 1 antibody. J Clin Oncol. 2019;37:2730-37.

11 Ishihara $\mathrm{H}$, Takagi $\mathrm{T}$, Kondo T, Homma C, Tachibana H, Fukuda H, et al. Association between immune-related adverse events and prognosis in patients with metastatic renal cell carcinoma treated with nivolumab. Urol Oncol. 2019;37(6):355.e21-e29.

12 Haratani K, Hayashi H, Chiba Y, Kudo K, Yonesaka K, Kato R, et al. Association of immune-related adverse events with nivolumab efficacy in non-small-cell lung cancer. JAMA Oncol. 2018;4(3):374-8.

13 Masuda K, Shoji H, Nagashima K, Yamamoto $\mathrm{S}$, Ishikawa $\mathrm{M}$, Imazeki $\mathrm{H}$, et al. Correlation between immune-related adverse events and prognosis in patients with gastric cancer treated with nivolumab. BMC Cancer. 2019; 19(1):974.

14 Das S, Ciombor KK, Haraldsdottir S, Pumpalova Y, Sahin IH, Pineda G, et al. Immunerelated adverse events and immune checkpoint inhibitor efficacy in patients with gastrointestinal cancer with food and drug administration-approved indications for immunotherapy. Oncologist. 2020;25(8):66979.

15 Horvat TZ, Adel NG, Dang TO, Momtaz P, Postow MA, Callahan MK, et al. Immune-related adverse events, need for systemic immunosuppression, and effects on survival and time to treatment failure in patients with melanoma treated with ipilimumab at Memorial Sloan Kettering Cancer Center. J Clin Oncol. 2015;33(28):3193-8.
16 Nigro O, Pinotti G, De Galitiis F, Di Pietro FR, Giusti R, Filetti M, et al. Late immune-related adverse events in long-term responders to PD-1/PD-L1 checkpoint inhibitors: a multicentre study. Eur J Cancer. 2020;134:19-28.

17 Petrelli F, Signorelli D, Ghidini M, Ghidini A, Pizzutilo EG, Ruggieri L, et al. Association of steroids use with survival in patients treated with immune checkpoint inhibitors: a systematic review and meta-analysis. Cancers. 2020;12(3):546.

18 Common Terminology Criteria for Adverse Events (CTCAE), Version 4.03. In: services USDoHaH, editor. United States of America, 2010.

19 Haanen JBAG, Carbonnel F, Robert C, Kerr KM, Peters S, Larkin J, et al. Management of toxicities from immunotherapy: ESMO clinical practice guidelines for diagnosis, treatment and follow-up. Ann Oncol. 2017; 28(suppl_4):iv119-42.

20 Brahmer JR, Lacchetti C, Schneider BJ, Atkins $\mathrm{MB}$, Brassil KJ, Caterino JM, et al. Management of immune-related adverse events in patients treated with immune checkpoint inhibitor therapy: American society of clinical oncology clinical practice guideline. J Clin Oncol. 2018;36(17):1714-68.

21 Dafni U. Landmark analysis at the 25-year landmark point. Circ Cardiovasc Qual Outcomes. 2011;4(3):363-71.

22 Grangeon M, Tomasini P, Chaleat S, Jeanson A, Souquet-Bressand M, Khobta N, et al. Association between immune-related adverse events and efficacy of immune checkpoint inhibitors in non-small-cell lung cancer. Clin Lung Cancer. 2019;20(3):201-7.

23 Ricciuti B, Genova C, De Giglio A, Bassanelli M, Dal Bello MG, Metro G, et al. Impact of immune-related adverse events on survival in patients with advanced non-small cell lung cancer treated with nivolumab: long-term outcomes from a multi-institutional analysis. J Cancer Res Clin Oncol. 2019;145(2):479-85.

24 Weber JS, Hodi FS, Wolchok JD, Topalian SL, Schadendorf D, Larkin J, et al. Safety profile of nivolumab monotherapy: a pooled analysis of patients with advanced melanoma. J Clin Oncol. 2017;35(7):785-92.

25 Foster CC, Kochanny S, Khattri A, Acharya R, Dekker A, Tan Y-HC, et al. Association of immune-related adverse events (irAEs) with improved response, progression-free survival, and overall survival for patients with metastatic head and neck cancer receiving antiPD-1 therapy. J Clin Oncol. 2018;36(15_suppl):6014.

26 Lutzky J, Wolchok J, Hamid O, Lebbe C, Pehamberger $\mathrm{H}$, Linette $\mathrm{G}$, et al. Association between immune-related adverse events (irAEs) and disease control or overall survival in patients (pts) with advanced melanoma treated with $10 \mathrm{mg} / \mathrm{kg}$ ipilimumab in three phase II clinical trials. J Clin Oncol. 2009;27(15_suppl):9034.
27 Attia P, Phan GQ, Maker AV, Robinson MR, Quezado MM, Yang JC, et al. Autoimmunity correlates with tumor regression in patients with metastatic melanoma treated with anticytotoxic T-lymphocyte antigen-4. J Clin Oncol. 2005;23(25):6043-53.

28 Passat T, Touchefeu Y, Gervois N, Jarry A, Bossard C, Bennouna J. [Physiopathological mechanisms of immune-related adverse events induced by anti-CTLA-4, anti-PD-1 and anti-PD-L1 antibodies in cancer treatment]. Bull Cancer. 2018;105(11):1033-41.

29 Shankar B, Zhang J, Naqash AR, Forde PM, Feliciano JL, Marrone KA, et al. Multisystem immune-related adverse events associated with immune checkpoint inhibitors for treatment of non-small cell lung cancer. JAMA Oncol. 2020;6(12):1952-6.

30 Quach HT, Dewan AK, Davis EJ, Ancell KK, Fan R, Ye F, et al. Association of anti-programmed cell death 1 cutaneous toxic effects with outcomes in patients with advanced melanoma. JAMA Oncol. 2019;5(6):906-8.

31 Arbour KC, Mezquita L, Long N, Rizvi H, Auclin $\mathrm{E}, \mathrm{Ni} \mathrm{A}$, et al. Impact of baseline steroids on efficacy of programmed cell death- 1 and programmed death-ligand 1 blockade in patients with non-small-cell lung cancer. J Clin Oncol. 2018;36(28):2872-8.

32 Pinato DJ, Kaseb A, Wang Y, Saeed A, Szafron $\mathrm{D}$, Jun $\mathrm{T}$, et al. Impact of corticosteroid therapy on the outcomes of hepatocellular carcinoma treated with immune checkpoint inhibitor therapy. J Immunother Cancer. 2020; 8(2): 0000726

33 El-Khoueiry AB, Sangro B, Yau T, Crocenzi TS, Kudo M, Hsu C, et al. Nivolumab in patients with advanced hepatocellular carcinoma (CheckMate 040): an open-label, noncomparative, phase $1 / 2$ dose escalation and expansion trial. Lancet. 2017;389(10088): 2492-502.

34 Zhu AX, Finn RS, Edeline J, Cattan S, Ogasawara S, Palmer D, et al. Pembrolizumab in patients with advanced hepatocellular carcinoma previously treated with sorafenib (KEYNOTE-224): a non-randomised, openlabel phase 2 trial. Lancet Oncol. 2018;19(7): 940-52.

35 Hurria A, Togawa K, Mohile SG, Owusu C, Klepin HD, Gross CP, et al. Predicting chemotherapy toxicity in older adults with cancer: a prospective multicenter study. J Clin Oncol. 2011;29(25):3457-65.

36 van Holstein Y, Kapiteijn E, Bastiaannet E, van den Bos F, Portielje J, de Glas NA. Efficacy and adverse events of immunotherapy with checkpoint inhibitors in older patients with cancer. Drugs Aging. 2019;36(10):92738.

37 Valpione S, Pasquali S, Campana L, Mocellin S, Piccin L, Pigozzo J, et al. Predictors of toxicity for metastatic melanoma patients treated with ipilimumab. J Immunother Cancer. 2015;3:P247. 
38 Irelli A, Sirufo MM, D’Ugo C, Ginaldi L, De Martinis M. Sex and gender influences on cancer immunotherapy response. Biomedicines. 2020;8(7):232.

39 Yao X, Li H, Leng SX. Inflammation and immune system alterations in frailty. Clin Geriatr Med. 2011;27(1):79-87.
40 Khaki AR, Li A, Diamantopoulos LN, Bilen MA, Santos V, Esther J, et al. Impact of performance status on treatment outcomes: a real-world study of advanced urothelial cancer treated with immune checkpoint inhibitors. Cancer. 2020;126(6):1208-16.
41 Petrillo LA, El-Jawahri A, Nipp RD, Lichtenstein MRL, Durbin SM, Reynolds KL, et al. Performance status and end-of-life care among adults with non-small cell lung cancer receiving immune checkpoint inhibitors. Cancer. 2020;126(10):2288-95.

42 Lu L, Xing K, Wei W, Ling Y, Li P, Li S, et al. Immune-related adverse events predict responses to PD-1 blockade immunotherapy in hepatocellular carcinoma. Int J Cancer. 2021 Apr 22. Online ahead of print. 